\title{
Calculation of Consumer and Manufacturer Risks for Unmanned Aerial Vehicle Checkout Equipment
}

\author{
N.F. Tupitsin \\ National Aviation University Aviation \\ Computer-Integrated \\ Complexes Department \\ Kyiv, Ukraine \\ nift@mail.ru
}

\author{
E.O. Krymov \\ National Aviation University Aviation \\ Computer-Integrated \\ Complexes Department \\ Kyiv, Ukraine \\ yegor.krymov@gmail.com
}

\author{
I.C. Stepanenko \\ National Aviation University Aviation \\ Computer-Integrated \\ Complexes Department \\ Kyiv, Ukraine \\ STEPANENKO.iLay@gMaiL.com
}

\begin{abstract}
The paper presents description of algorithms for calculation values of consumer and manufacturer risks. Determination of UAV technical state with the account of received risks reflects the information about how much more it will work.
\end{abstract}

Keywords-consumer risk; manufacturer risk; checkout equipment; controlled object; controlled parameters

\section{INTRODUCTION}

It is known from the practice that a risk is accompanied by any business. In the sphere of UAV production and exploitation, the risks appear on stage of transmission UAV from manufacturer to consumer and after that manifest every time at checking its technical state [1], [2].

Timely calculation and analyses of all possible risks help to minimize their negative consequences on the manufacturer of UAV. It is assumed in the sequel that errors of production lead to the fact that all controlled parameters (CP) of the checking object $(\mathrm{CO})$ are distributed according to the normal law.

The manufacturer risk $(A)$ represents the probability of culling of a $\mathrm{CO}$, due to poor or pessimistic $\mathrm{CP}$, even if in reality it is corresponded acceptable level.

The consumer risk $(B)$ is the probability of a CO acceptance due to the random favorable sample while the overall quality of products worst than the acceptable level.

Development of the main characteristics of the UAV checking algorithm is not a separate, insulated task, and it needs to be considered in the context of the effective implementation of the tasks standing before the UAV. Support of maintenance and metrological control of checkout equipment (CE) for UAV is the independent task. One of the most important indicators of functioning of the CE is values $A$ and $B$. At increasing the values of $A$ and $B$, the likelihood of using inoperative $\mathrm{CO}$ is raised. The likelihood of culling workable $\mathrm{CO}$ is raised also. This is especially important for such type of product as expensive UAV. The maintaining of given level of UAV performance one may provide due to the proper organization of the volume and frequency of inspections of their basic characteristics.

\section{Problem Statement}

In connection with the rapid development of the UAV fleet and increasingly wide of their use the task of maintaining uptime these UAVs is becoming very urgent. For the improvement of intensity and safety of the flights, should be decreased downtime of aeronautical engineering and increase the reliability information about its technical state. The problem can be solved by the way of design and implementation into exploitation progressive methods of the checking of aeronautical engineering.

Well known that the task of UAV service includes not only detecting and repairing the failing UAV, but also maximal decreases the number of refusals of the $\mathrm{CO}$ by the way of right organization of the number and volume of equipment checking. It can be solved by using methods of prognosis of the technical state of the object. So, the problem of providing the receiving the high effectiveness of aeronautical engineering, in part is solved with help of equipment, which executes the effective control of the technical state of $\mathrm{CO}$.

For improvement of the methods of control and diagnostics use the newest advancements in the methods of automated checking of the UAV.

At the aeronautical engineering there is an opportunity to use the complexes of automated control and diagnostics, in which composition is the checkout equipment.

The block diagram of the interaction of the control object and the checkout equipment is shown in Fig. 1.

Checking the precision of subsystems and devices CE conducted either under periodic maintenance or in the process of restoring a failed $\mathrm{CE}$. The increase in volume and a reduction of a period between inspections lead to an increase in the UAV service cost and a reduction of the time, which the UAV remains in operating condition. However, in this case, the losses are reduced of UAV associated with the adoption of wrong decisions it's checking. And therefore it is advisable to consider the problem of choosing the volume and frequency of checks in a more general setting.

Suppose that there are $N$ objects of the same type of checking with the known laws of distribution of controlled parameters $f_{i}(v), i=1, n$; tolerances on the parameters $\left(a_{i}, b_{i}\right)$. 
For checking of UAV are used the $k$ channels of control (CC). From $k$ channels of control $k 1$ subject to periodic verification through a period $T_{k}$. The laws of distribution of CC error $\varphi_{j}(\Delta), j=1, . ., k_{1} ; \Delta_{V j}, \Delta_{H j}$ are upper and lower allowable values of error, respectively.

It is necessary to define such values $k_{1}$ and $T_{k}$, at which the sum of maintenance costs $\mathrm{CE}$ and $\mathrm{CO}$ is minimal at given restrictions on risks $\mathrm{A}$ and $\mathrm{B}$

$$
R=\min F_{1}\left(k_{1}, T_{K}\right), \text { at } A \leq A_{\mathrm{g}}, \quad B \leq B_{\mathrm{g}},
$$

where $F_{1}$ is the sum of operating costs for the $\mathrm{CO}$ and the sum of operating costs for the CE; $A_{\mathrm{g}}$ is the given value $A$, $B_{\mathrm{g}}-$ given value $B$.

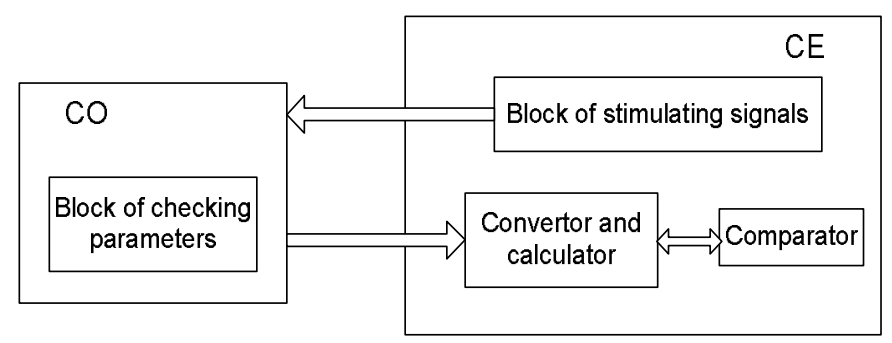

Fig. 1. The block diagram of the interaction between $\mathrm{CO}$ and $\mathrm{CE}$.

\section{Algorism of Defining the TeChNicAL AND ECONOMICAL EFFICIENCIES OF DIAGNOSTICS}

\section{A. Technical index of diagnostics efficiency}

Expedience of verification of the technical state of object is estimated by the indexes of diagnostics efficiency. These indexes are defined by comparison of benefit which is got in the result of conducting of periodic diagnostics, with costs on it conducting.

An increase of probability of fitness of object is in the case of introduction of periodic control

$$
\Delta P=P_{1}-P_{2}
$$

where $P_{1}$ is the probability of object fitness during periodical diagnostics,

$$
P_{1}=\frac{P-A}{P-A+B} e^{-\omega T}
$$

where $T$ is the period of object diagnostics; $P_{2}$ is the probability of object fitness at the absence of diagnostics during life duration,

$$
P_{2}=\frac{P-A}{P-A+B} e^{-\omega t}
$$

where $0<t<T_{P D}\left(T_{P D}\right.$ is the life duration); $\omega$ is the parameter of failure flux.

\section{B. Economic index of diagnostics efficiency}

The value of an economic index of diagnostics efficiency.

$$
\Delta C=C_{1}-C_{2},
$$

where $C_{1}$ is a cost of objects, economized due to diagnostics, which is determined a difference between the amount of undiagnosed and amount of diagnosed objects, necessary for the fulfilment of the same task (the undiagnosed are needed more than diagnosed), thus

$$
C_{1}=C_{C} N_{0}\left[\frac{\ln \left(1-P_{1}\right)}{\ln \left(1-P_{2}\right)}-1\right],
$$

where $C_{C}$ is the controlled object cost; $N_{0}$ is the quantity of objects; $C_{2}$ is the cost of diagnostics and maintenance of objects with periodical diagnostics.

Value of $C_{2}$ is calculated by the formula

$$
C_{2}=C_{C E}+\left(C_{R}+C_{C D C}+C_{E E}\right) N_{0} T_{O E}
$$

where $T_{O E}$ is current time of object exploitation; $C_{C E}$ is the cost of $C E ; C_{R}$ is the expenditures on CE renew

$$
C_{E R}=\frac{C_{T C} T_{D D C}}{T_{T R}},
$$

where $C_{T C}$ is the total cost on renew of $\mathrm{CE}$ during life duration; $T_{D D C}$ is the duration of one diagnostics cycle; $T_{T R}$ is the technical resource of $\mathrm{CE} ; C_{C D C}$ is the cost of deterioration of object during one diagnostics cycle

$$
C_{C D C}=\frac{C_{C} T_{D D C}}{T_{T A}},
$$

where $T_{T A}$ is the technical age of object.

Cost of exploitation expenditures $C_{E}$ during one diagnostics cycle

$$
C_{E}=C_{A C} T_{D D C}
$$

where $C_{A C}$ is the average cost of exploitation expenditures per one hour.

So, equations (2) - (4) allow to calculate the technical index of diagnostics efficiency and equations (5) - (10) is the economic index of diagnostics efficiency.

\section{Algorism of Defining Risks}

Risks of manufacturer $(\alpha)$ and customer $(\beta)$ for one control led parameter by using one testing channel are calculated in form [3]: 


$$
\begin{gathered}
\alpha=\int_{a}^{b} f(V)\left[\int_{-\infty}^{a-V} \Psi(\xi) d \xi+\int_{b-V}^{\infty} \Psi(\xi) d \xi\right] d V, \\
\beta=\int_{-\infty}^{a} f(V) \int_{a-V}^{b-V} \Psi(\xi) d \xi d V+\int_{b}^{\infty} f(V) \int_{a-V}^{b-V} \Psi(\xi) d \xi d V,
\end{gathered}
$$

where $f(V)$ is the distribution density of the control parameter; $\Psi(\xi)$ is the distribution density of the checking inaccuracy; $a, b$ are lower and upper limits of tolerance of controllable parameter.

If $P_{i}$ is a priori probability of the able-bodied condition of the $\mathrm{CO}$ on $i$ th controlled parameter; $\alpha_{i}, \beta_{i}$ is the risks of the producer and the customer on $i$ th controlled parameter; $N$ is an amount controlled parameters of the $\mathrm{CO}$, that total risks of the producer $A$ and customer $B$, as well as generalized factor to validity of the checking $D$ are found with help of formulas:

$$
\begin{aligned}
A & =\prod_{i=1}^{N} P_{i}-\prod_{i=1}^{N}\left(P_{i}-\alpha_{i}\right) \\
B & =\prod_{i=1}^{N}\left(P_{i}-\alpha_{i}+\beta_{i}\right)-\prod_{i=1}^{N}\left(P_{i}-\alpha_{i}\right) \\
D & =1-A-B
\end{aligned}
$$

The expressions (11) - (12) are complex at calculation many times in process of CO testing or self-testing of CE. Therefore was developed simpler analytical algorism for quick calculation risks of manufacturer and customer.

Assume that we have equipment of checkout with $K 1$ channels of control. Each CC may be in good state with probability $P_{\kappa}^{(1)}$ and failed state - with a probability $P_{\kappa}^{(2)}$. To obtain of dependencies $A$ and $B$ we consider the graph of control (Fig. 2).

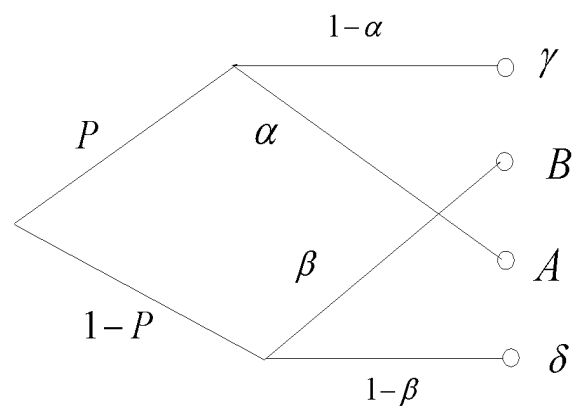

Fig. 2. The graph of control, where $P$ is a probability that the CO in good condition before control; $\gamma$ is the probability of making the right decision "suitable"; $\delta$ is the probability of making the right decision "unsuitable"; $\alpha, \beta$ are type I and type II errors under the control of CO, respectively.

On basis of the graph of control we can write

$$
A=P-\gamma
$$

In case of independence of $\mathrm{CC}$

$$
\gamma=\prod_{j=1}^{k_{1}} \gamma_{j}
$$

where $\gamma_{j}$ is the probability of making the right decision "suitable " at testing on $j$ th CC.

The probability of a correct decision "suitable" under the control of the $j$ th $\mathrm{CC}$ defined as the probability of a correct decision "suitable" for all $n_{j}$ parameters, which are controlled by these $\mathrm{CC}$, i.e.

$$
\gamma_{j}=\sum_{l=1}^{2} P_{k}^{(l)} \prod_{i=1}^{n j}\left(P_{i}-\alpha_{i j}^{(l)}\right)
$$

where $P_{i}$ is a priori probability of right solution relatively the $i$ th parameter of $\mathrm{CO}, \alpha_{i j}^{(l)}$ is the risk of the manufacturer of the $i$ th parameter $\mathrm{CO}$, which is controlled by a $j$ th parameter of $\mathrm{CC}$, located in $l$ th state.

Substituting (16) in (15) with account (14), we obtain formula for the producer's risk

$$
A=P-\prod_{j=1}^{k_{1}}\left(\sum_{l=1}^{2} P_{k}^{(l)} \prod_{i=1}^{n j}\left(P_{i}-\alpha_{i j}^{(l)}\right)\right) .
$$

Similarly, we obtain expressions for the customer risk.

Equations for calculations of $A$ and $B$ are given for $k_{1}$ independent $\mathrm{CC}$, each of which may be operative or inoperative states.

Risks $\alpha_{\mathrm{ij}}{ }^{(1)}, \beta_{\mathrm{ij}}{ }^{(1)}$ on the $i$ th controllable parameters are calculated by different formulas depending on the status of CC checkout equipment. If the errors in the controls tolerance limits $(l=1)$ then the expression of risk has next type

$$
\alpha_{i j}^{(1)}=\frac{1}{P_{k j}^{1}}(J 1+J 2),
$$

where $J 1=\int_{a_{i}}^{a_{i}-\Delta_{H j}} \int_{\Delta_{H j}}^{a-v_{i i}} f_{i}\left(v_{i}\right) \phi_{j}\left(\Delta_{j}\right) d v_{i} d \Delta_{j}$;

$$
J 2=\int_{b_{i}-\Delta_{B_{j}}}^{b_{i}} \int_{b_{j}-v}^{\Delta_{B_{j}}} f_{i}\left(v_{i}\right) \phi_{j}\left(\Delta_{j}\right) d v_{i} d \Delta_{j}
$$

$$
\beta_{i j}^{(1)}=\frac{1}{P_{k j}^{1}}(J 3+J 4),
$$

where $J 3=\int_{a_{i}-\Delta_{B j}}^{a_{i}} \int_{a_{i}}^{\Delta_{B j}} f_{i}\left(v_{i}\right) \phi_{j}\left(\Delta_{j}\right) d v_{i} d \Delta_{j}$; 


$$
J 4=\int_{\Delta_{H j}}^{b_{i}-v_{i}} \int_{\Delta_{H_{j}}}^{b_{i}-v_{i}} f_{i}\left(v_{i}\right) \phi_{j}\left(\Delta_{j}\right) d v_{i} d \Delta_{j}
$$

where $a_{i}, b_{i}$ are the lower and upper limits of tolerance of $i$ th controllable parameter ; $\Delta_{H j}, \Delta_{B_{j}}$ are the lower and upper limits of tolerance of error of $j$ the checking channel; $f_{i}\left(v_{i}\right)$ is the normal probability density of $i$ th controllable parameter; $\phi_{j}\left(\Delta_{j}\right)$ is the normal probability density of error of $j$ th checking channel.

If you find that errors testing out of tolerance $(l=2)$ then

$$
\alpha_{i j}^{(2)}=\frac{1}{P_{k j}^{1}}(J 5+J 6)+\Delta \alpha,
$$

where $J 5=\int_{a_{i}}^{a_{i}-\Delta_{H j}} \int_{-\infty}^{\Delta_{H j}} f_{i}\left(v_{i}\right) \phi_{j}\left(\Delta_{j}\right) d v_{i} d \Delta_{j}$

$J 6=\int_{a_{i}-\Delta_{B j}}^{b_{i}} \int_{-\infty}^{a_{i}-v_{i}} f_{i}\left(v_{i}\right) \phi_{j}\left(\Delta_{j}\right) d v_{i} d \Delta_{j} ; \Delta \alpha=\frac{1}{P_{k j}^{1}}(J 7+J 8)$,

where $J 7=\int_{a_{i}}^{b_{i}-\Delta_{B j}} \int_{b_{i}-v_{i}}^{\infty} f_{i}\left(v_{i}\right) \phi_{j}\left(\Delta_{j}\right) d v_{i} d \Delta_{j}$;

$J 8=\int_{b_{i}-\Delta_{B j}}^{b_{i}} \int_{\Delta_{B j}}^{\infty} f_{i}\left(v_{i}\right) \phi_{j}\left(\Delta_{j}\right) d v_{i} d \Delta_{j}$

$$
\beta_{\mathrm{ij}}^{(2)}=\frac{1}{1-P_{k j}^{1}}(J 9+J 10)+\Delta \beta
$$

where $J 9=\int_{-\infty}^{a_{i}-\Delta_{B j}} \int_{a_{i}-v_{i}}^{b_{i}-v_{i}} f_{i}\left(v_{i}\right) \phi_{j}\left(\Delta_{j}\right) d v_{i} d \Delta_{j}$;

$J 10=\int_{a_{i}-\Delta_{B j}}^{a_{i}} \int_{\Delta_{B_{j}}}^{b_{i}-v_{i}} f_{i}\left(v_{i}\right) \phi_{j}\left(\Delta_{j}\right) d v_{i} d \Delta_{j} ; \Delta \beta=J 11+J 12$,

where $J 11=\int_{b_{i}}^{b_{i}-\Delta_{H j}} \int_{a_{i}-v_{i}}^{\Delta_{B j}} f_{i}\left(v_{i}\right) \phi_{j}\left(\Delta_{j}\right) d v_{i} d \Delta_{j}$;
$J 12=\int_{b_{i}-\Delta_{H j}}^{\infty} \int_{a_{i}-v_{i}}^{b_{i}-v_{i}} f_{i}\left(v_{i}\right) \phi_{j}\left(\Delta_{j}\right) d v_{i} d \Delta_{j}$.

Obtained formulas can calculate numerically only.

If information on a priori probability of the able-bodied condition of the CO for each controlled parameter $\left(P_{i}\right)$ is absent, but there is information on a priori probability $\left(P_{a}\right)$ of the $\mathrm{CO}$, in general, then possible to estimate the value $P_{i}$ by formula:

$$
P_{i}=\sqrt[m]{P_{a}}
$$

where $m$ is an amount defining parameters of the CO.

The expressions (17) - (21) may be used in process of $\mathrm{CO}$ testing or self-testing of CE.

\section{CONCLUSION}

In this paper the algorithm of defining the technical and economical efficiencies of UAV diagnostics is submitted. One of the essential difficulties at realization this algorism is a task to calculate the consumer and manufacturer risks.

The algorithm and program in programming environment Delphi for calculating values of consumer and manufacturer risks were developed. Using of the developed algorithm and program allows receiving the correct information about the technical state of control object and give prognoses about its future work.

In addition, the algorithm for calculation approximate values of consumer and manufacturer risks with help of analytical formulas was developed [4].

\section{REFERENCES}

[1] A.A. Gorovoj, V.F. Vaschevsky, and B.I. Dotsenko, Microprocessor aggregate complexes for diagnosing technical systems ", Kyiv, Technique, 1990, 168 p. (in Russian)

[2] S.N. Zimin, M.V. Ulanov, and V.A. Smirnov. "The process of technical control and diagnostics systems on board unmanned aircraft to support decision-making and complex test equipment with intelligent decision support system for its implementation”. Russian Patent № 2557771. Moscow, 2014. (in Russian).

[3] R.N. Belokon, V.N. Kozak., and G.P. Cherny, Reliability of selfchecking, Daugavpils: DHMAIU, 1988, 57 p. (in Russian)

[4] G.P. Cherny, V. I. Tarasenko, and M.F. Tupitsyn, Automated control system. Guide for Laboratory works. Kyiv, NAU, 2006, 48 p. (in Russian) 